\title{
Primary Sclerosing Cholangitis in an Elderly Patient: A Diagnostic Challenge
}

\author{
Tobar-Marcillo Marco ${ }^{1,2}$, Vela-Vizcaino Hiram ${ }^{1,2}$, Pliego-Reyes Lenin ${ }^{1,2}$ \\ ${ }^{1}$ Department of Internal Medicine, Regional Hospital "Licenciado Adolfo Lopez Mateos, Institute for Social Security and Services for State \\ Workers", Mexico City, ${ }^{2}$ National Autonomous University of Mexico, School of Medicine, Mexico City, Mexico
}

Corresponding Author:

Tobar-Marcillo Marco

National Autonomous University of Mexico,

School of Medicine, Avenida Gabriel Mancera

1258, Colonia del Valle, Delegation Benito

Juarez, Mexico City 03100, Mexico

Tel: $+521-5566540252$

Fax: $+521-56614469$

E-mail: marcotobar1@hotmail.com

Received: May 24, 2017

Revised: September 22, 2017

Accepted: September 22, 2017

\begin{abstract}
Jaundice syndrome is a diagnostic challenge for physicians. It must be diagnosed based on its etiology, which depends on liver function test results, clinical manifestations, and age of presentation, and the objective is to rule out the most common causes and then the rare causes. We describe the case of a male patient aged 86 years who had jaundice and was diagnosed as having primary sclerosing cholangitis based on liver biopsy findings. It is a chronic, progressive cholestatic liver disease with unknown etiology until now. Primary sclerosing cholangitis is characterized by inflammation, fibrosis, and stenosis of small, medium, and large intrahepatic and extrahepatic biliary ducts. Although it is a rare entity in geriatric patients, certain characteristics are infrequently considered in clinical practice; thus, considering all characteristics of this disease would help physicians make the diagnosis earlier. It is a condition that should be considered when jaundice is associated with pruritus and elevated levels of peripheral eosinophils and immu noglobulin $\mathrm{E}$.
\end{abstract}

Key Words: Cholangitis, Sclerosing, Jaundice, Elderly

\section{INTRODUCTION}

Primary sclerosing cholangitis (PSC) is defined by chronic, progressive cholestatic liver disease with an unknown etiology until now. It is characterized by inflammation, fibrosis, and stenosis of small, medium, and large intrahepatic and extrahepatic biliary ducts ${ }^{1}$. With an incidence rate of 0.77 per 100,000 person-years, PSC is more common in men than in women at a ratio of 1.7:1.

The mean age at diagnosis of PSC is approximately 40 years for European and North America populations; however, it is uncommon in patients older than 50 years. Japan is the only country that reported a second incidence peak in individuals aged $50-60$ years $^{2)}$. It was first described in 1924 by Delbet, and it is associated with multiple complications, such as biliary tract stenosis, cholelithiasis, cholangitis, cholangiocarcinoma, and colon cancer in patients with concomitant ulcerative colitis".

Since it is a rare clinical entity in geriatric individuals, we describe an 86-year-old patient who was found to have PSC within the management protocol of cholestatic jaundice. Interestingly, some features observed during the presenta- tion and evolution of PSC in our patient indicated that this disease manifests differently in young patients.

\section{CASE REPORT}

An 86-year-old man had a history of smoking (less than one pack per day for 4 years) and a habit of consuming liquor every 2 months, which he quit 5 years ago. He also had a history of syphilis infection twice during his youth that was eradicated with benzathine penicillin. He had undergone radical prostatectomy due to prostatic hyperplasia. Additionally, he was diagnosed as having prediabetes, which was controlled with biguanides, 10 months before his admission.

He presented with a 40-day disease evolution predominantly characterized by nocturnal itching, jaundice, and dark urine. Laboratory tests on admission showed the following results: total bilirubin (TB) level, $17.2 \mathrm{mg} / \mathrm{dL}$ (reference range, $0.5-1.3 \mathrm{mg} / \mathrm{dL}$ ); direct bilirubin level, $13.1 \mathrm{mg} / \mathrm{dL}$; alkaline phosphatase level, $158 \mathrm{U} / \mathrm{L}$ (reference range, 50-140 U/L); gamma glutamyl transpeptidase level, 109.8 U/L (reference range, 5-85 $\mathrm{U} / \mathrm{L}$ ); alanine aminotransferase level, $46 \mathrm{U} / \mathrm{L}^{\text {; }}$ aspartate aminotransferase (AST) level, $34 \mathrm{U} / \mathrm{L}$ (reference range, 24-36 U/L); and serum albumin level, $2.8 \mathrm{gr} / \mathrm{dL}$. There was a 
slight increase in the level of eosinophils, he was negative for viral hepatitis, and his tumor antigen level was within normal range.

The ultrasonogram of the liver and biliary tract showed the Chilaiditi sign, which consists of overlapping of the intestine between the liver and right diaphragm, and has an incidence of $0.4 \%-12 \%$. The ultrasonogram also showed biliary tract dilation, a 5-mm bile duct, hepatic parenchyma with increased echogenicity probably related to the inflammatory process, an 8-mm portal vein, and obscured gallbladder. The abdominal computed tomography scan showed no specific findings. To achieve better bile duct appreciation, endoscopic retrograde cholagiopancreatography (ERCP) was performed, and a lesion was found with normal macroscopic features. Fluoroscopy showed 10-mm dilatation of the main bile duct without any defects in filling and an intrahepatic duct without any dilation, and the magnetic resonance cholangiogram showed no defects in filling. In summary, only a bile duct was slightly dilated, with regular, smooth edges of the biliary duct, and a lesion of the ampulla of Vater

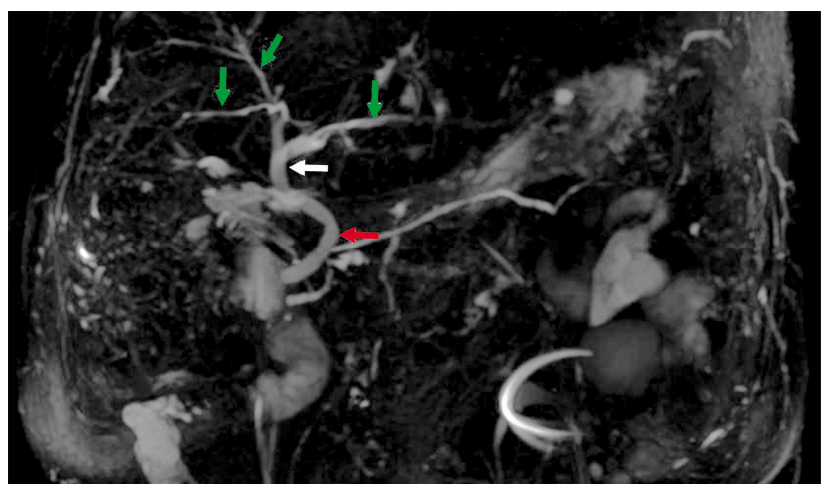

Fig. 1. Colangioresonance phase T1 showing biliary tree integrity, intrahepatic ducts without obliterations (green arrows); common hepatic duct with diameter of $8.9 \mathrm{~mm}$, no obstruction signs (white arrow); bile duct without the presence of obliteration and bile duct diameter of maximum $9.11 \mathrm{~mm}$ (red arrow). was found with normal tone characteristics and without extrinsic compression (Fig. 1). Thus, we continued our diagnostic protocol. The patient was negative for mitochondrial antibodies and perinuclear antineutrophil cytoplasmic antibodies; had normal levels of $\mathrm{lgG}(962 \mathrm{mg} / \mathrm{dL}$; reference range, $700-1,600 \mathrm{mg} / \mathrm{dL})$ and $\mathrm{lgG} 4(7.16 \mathrm{mg} / \mathrm{dL}$; reference range, $3.0-200 \mathrm{mg} / \mathrm{dL})$; had an increasing lgE level $(328 \mathrm{IU} / \mathrm{mL}$; reference range, $0-200 \mathrm{lU} / \mathrm{mL}$ ); and was negative for antinuclear autoantibodies, antismooth muscle antibodies, and liver kidney microsomal type 1 antibodies. The aforementioned results led us to overlook the presence of autoinmune hepatitis. Since the diagnosis was still unclear, we decided to perform an ultrasound-guided biopsy of the liver. We observed mononuclear inflammatory infiltrates in the small caliber ducts, concentric fibrosis with an onion skin pattern, and hyperplasia of Kupffer cells. All these data were associated with the clinical presentation and characteristic laboratory findings of PSC of the small bile duct (Fig. 2). Ultimately, PSC was diagnosed, and the patient had a Mayo risk score of 3.54. He had estimated survivals of 1 year (64\% chance) and 4 years (10\% chance).

Symptomatic treatment was started with loratadine $(10 \mathrm{mg}$ orally every 24 hours) and ursodeoxycholic acid (300 mg orally every 8 hours). Follow-up laboratory test results were as follows: TB level, $13.4 \mathrm{mg} / \mathrm{dL}$; seric albumin level, $2.5 \mathrm{~g} / \mathrm{dL}$; and AST level, $62 \mathrm{U} / \mathrm{L}$. Endoscopy showed esophageal varices in the distal third of the esophagus. The patient has had no variceal bleeding with a Mayo risk score of 4.05. After 1 year of treatment, he remains stable and shows no significative progression. Fig. 3 shows the clinical course of the disease at the 1-year follow-up based on the parameters established by the Mayo risk score.

The authors state that has followed the protocols his workplace on patient data publication. Right to privacy and informed consent. The authors have obtained patient's informed consent referred to in Article. This document work in the power of the corresponding author.
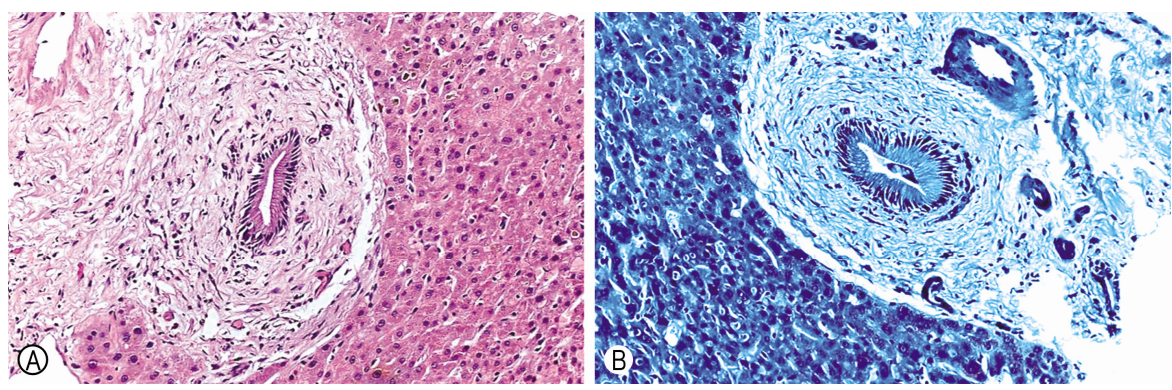

Fig. 2. Cutting photomicrograph liver: hematoxidin eosin staining, $\times 40(\mathrm{~A})$, Methylene blue staining, $\times 40$ (B). Where shows portal tracts atrophic or surrounded by concentric fibrosis, implicating the entire portal space was done; the branches of the portal vein were dilated, vein center lobular unchanged, the sinusoids show hyperplasia Kupffer cells that have phagocytosed, negative hemosiderin orcein staining. 


\section{DISCUSSION}

PSC predominantly occurs in people aged between 37 and 43 years ${ }^{2)}$. Its epidemiological charcterisics lead to a higher disease prevalence in the fourth and fifth decades of life. A meta-analysis of data included from eight studies in Europe and North America reported that only one patient aged more than 84 years was diagnosed as having this disease $^{2,3)}$; however, Japan is the only country that reported a second peak of incidence in the sixth decade of life. In its latest revision, the study found that 3 of 388 patients

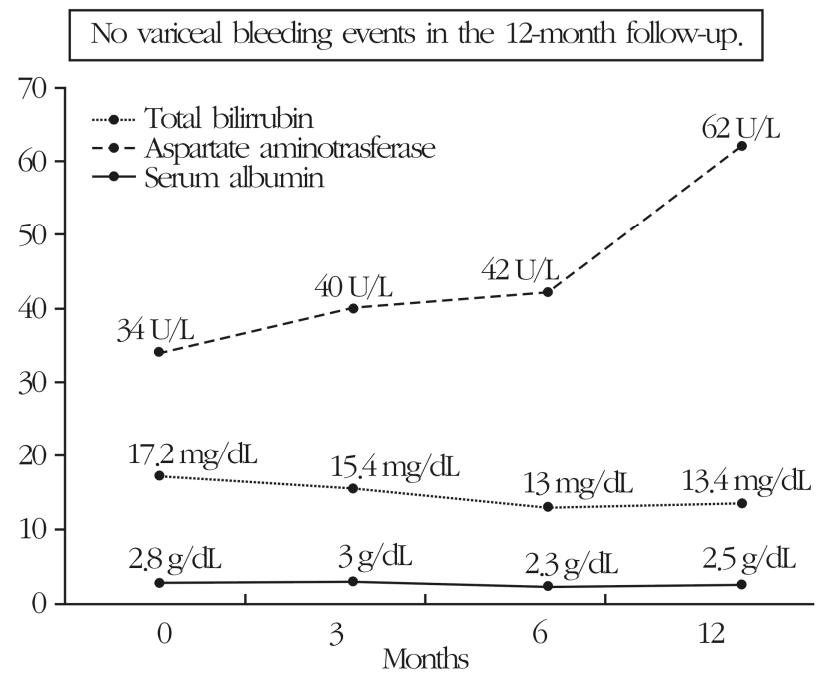

Fig. 3. The description of the 1-year follow-up diagnosis of primary sclerosing cholangitis, taking into account the criteria assessed by the Mayo Risk Score, which maintains it at high risk but without presenting a significant deterioration. with PSC were older than 80 years ${ }^{4)}$.

The description of clinical and laboratory features of PSC available in the literature do not differ according to age at diagnosis. There is only one study in which patients were classified according to age (more than or less than 50 years) ${ }^{4)}$. The cause of PSC is unknown, and three possible mechanisms have often been proposed for its pathogenesis, which include autoimmune diseases, chronic bacterial entry to the biliary tree, and ischemic damage of the bile ducts ${ }^{5}$. One of the most common laboratory findings is an increased lgM level in about $50 \%$ of patients. However, it has been reported that older patients do not present with this laboratory finding frequently; instead, a slightly elevated lgE level ${ }^{6)}$ and eosinophilia $^{7)}$ is observed.

Among the characteristics described in older patients, there is an association between PSC and inflammatory bowel disease, particularly ulcerative colitis is present in 10\% of older patients ${ }^{6)}$ compared to $90 \%$ in younger patients ${ }^{1)}$. However, this association was not found in the present patient, suggesting that the disease has different pathophysiological mechanisms depending on the age of presentation.

Regarding the analysis of antibodies, the most common finding of PSC is an increased level of antibodies against atypical perinuclear cytoplasm of neutrophils. However, 30\%$80 \%$ of patients ${ }^{8)}$ were reported as being negative for these antibodies, and $8 \%-77 \%$ of patients were positive for antinuclear antibodies, with a slight elevation and homogeneous pattern. Furthermore, positivity of human leukocyte antigen DRW52a is present in $0 \%-100 \%$ of patients with PSC ${ }^{9)}$, and the lgG4 level is estimated to be positive in $9 \%$; yet, the presence of other antibodies is not well established to have a clinical significance, including smooth muscle antibodies against biliary epithelium cells, antiperoxidase, etc. An impor-

Table 1. Summary of the differences and similarities in the presentation of PSC between young and elderly patients

\begin{tabular}{|c|c|}
\hline Variable & Summary \\
\hline Clinical presentation & Itching and subsequent appearance of jaundice appear to be the predominant clinical signs in all patients. \\
\hline \multicolumn{2}{|l|}{ Sex } \\
\hline Young patients & Predominately in men with a close relationship of $2: 1$ \\
\hline Elderly patients & Slightly more predominant in women \\
\hline \multicolumn{2}{|l|}{ Ulcerative colitis } \\
\hline Young patients & $90 \%$ of patients. \\
\hline Elderly patients & $10 \%$ of patients. \\
\hline Location & Predominately in the intrahepatic and extrahepatic bile ducts. \\
\hline \multicolumn{2}{|l|}{ Ig levels } \\
\hline Young patients & Increased IgM level by up to 50 . \\
\hline Elderly patients & Increased IgE level by about 87.5 . \\
\hline \multicolumn{2}{|l|}{ Antinuclear antibodies } \\
\hline Young patients & $30 \%$ of patients \\
\hline Elderly patients & $0 \%$ of patients \\
\hline
\end{tabular}


tant point to mention is that the positive data in various antibodies are not related to the severity of the disease or age of presentation ${ }^{8)}$.

Itching is the main symptom in patients with PSC, which is not related to the bilirubin level. It is rare to find elevation of more than $10 \mathrm{mg} / \mathrm{dL}$, besides typical laboratory features on presentation such as increased levels of alkaline phosphatase and transpeptidase gamma glutamyl ${ }^{1}$. However, this feature was not presented in our patient, which drew attention to the significant increase in the bilirubin level and slightly elevated alkaline phosphatase level.

Diagnostic confirmation of PSC is performed by imaging studies, mainly magnetic resonance cholangiography or ERCP, where multifocal structures and dilatation of the intrahepatic bile ducts and/or extrahepatic are shown, with a sensitivity of $86 \%$ and specificity of $94 \%$, respectively ${ }^{10)}$. Such features are generally seen in $89 \%$ of patients with extrahepatic duct involvement associated with intrahepatic ductal involvement. Eleven percent of patients with PSC only present with a defect of the small bile duct, so the diagnosis is established by performing a liver biopsy. The most specific finding is fibrous obliteration of the small bile ducts, with replacement by concentric connective tissue with an onion skin pattern, although this histological finding is observed in less than $25 \%$ of liver biopsies.

Histological abnormalities of PSC are nonspecific and similar to those of primary biliary cirrhosis; thus, they are discounted because of the absence of antimitochondrial antibodies that are present in 95\% of patients with PSC ${ }^{11,12)}$. One study reported that the pathology affecting intrahepatic ducts occurs in older patients with an average of 39.9 versus 32.5 years at diagnosis ${ }^{13)}$. In addition, patients with PSC who have a condition of the intrahepatic duct have a better prognosis and longer survival with less progression to cholangiocarcinoma compared to PSC patients with involvement of the extrahepatic ducts $(0 \% \text { vs. } 11 \%)^{14)}$.

The age of presentation plays an important role in prognosis since PSC is associated with malignant diseases, including cholangiocarcinoma, affecting up to $38 \%$ of younger patients versus $10 \%$ of geriatric patients. As the association with inflammatory bowel disease decreases the risk of colon cancer, it seems that older patients have a better prognosis in terms of morbidity and mortality ${ }^{6}$. This differs from the risk score established by the Mayo Clinic $^{15)}$ and validated for this purpose; older age is associated with a worse prognosis. In Table 1 summarizes differences and similarities comparison in the presentation of CEP in young and elderly patients.

In conclusion, jaundice syndrome is a diagnostic challenge for physicians. It must be diagnosed based on its etiology, which depends on the liver function test results, clinical manifestations, and age of presentation. Although PSC is a rare entity in geriatric patients, there are certain characteristics that differentiate it from the way in which it manifests in young patients. We intended to emphasize the characteristics that are infrequently considered in clinical practice, i.e., mainly an elevation of eosinophils and IgE level, and absence of antinuclear antibodies and ulcerative colitis, to help physicians make an early diagnosis of PSC.

Conflicts of Interest Disclosures: The researchers claim no conflicts of interest.

\section{REFERENCES}

1. Lindor KD, Kowdley KV, Harrison ME; American College of Gastroenterology. ACG clinical guideline: primary sclerosing cholangitis. Am J Gastroenterol 2015;110:646-59.

2. Molodecky NA, Kareemi H, Parab R, Barkema HW, Quan H, Myers RP, et al Incidence of primary sclerosing cholangitis: a systematic review and meta-analysis. Hepatology 2011;53:1590-9.

3. Card TR, Solaymani-Dodaran M, West J. Incidence and mortality of primary sclerosing cholangitis in the UK: a populationbased cohort study. J Hepatol 2008;48:939-44.

4. Takikawa H, Takamori Y, Tanaka A, Kurihara H, Nakanuma Y. Analysis of 388 cases of primary sclerosing cholangitis in Japan; Presence of a subgroup without pancreatic involvement in older patients. Hepatol Res 2004;29:153-9.

5. Lee YM, Kaplan MM. Primary sclerosing cholangitis. N Engl J Med 1995;332:924-33.

6. Hirano K, Tada M, Isayama H, Yashima Y, Yagioka H, Sasaki $\mathrm{T}$, et al. Clinical features of primary sclerosing cholangitis with onset age above 50 years. J Gastroenterol 2008;43:729-33.

7. Ichikawa $\mathrm{N}$, Taniguchi A, Akama $\mathrm{H}$, Ishiguro $\mathrm{H}$, Kurihara $\mathrm{T}$, Terai C, et al. Sclerosing cholangitis associated with hypereosinophilic syndrome. Intern Med 1997;36:561-4.

8. Angulo P, Peter JB, Gershwin ME, DeSotel CK, Shoenfeld Y, Ahmed AE, et al. Serum autoantibodies in patients with primary sclerosing cholangitis. J Hepatol 2000;32:182-7.

9. Prochazka EJ, Terasaki PI, Park MS, Goldstein LI, Busuttil RW. Association of primary sclerosing cholangitis with HLADRw52a. N Engl J Med 1990;322:1842-4.

10. Dave M, Elmunzer BJ, Dwamena BA, Higgins PD. Primary sclerosing cholangitis: meta-analysis of diagnostic performance of MR cholangiopancreatography. Radiology 2010;256:387-96.

11. Burak KW, Angulo P, Lindor KD. Is there a role for liver biopsy in primary sclerosing cholangitis? Am J Gastroenterol 2003;98: 1155-8.

12. Miyakawa H, Tanaka A, Kikuchi K, Matsushita M, Kitazawa E, Kawaguchi N, et al. Detection of antimitochondrial autoantibodies in immunofluorescent AMA-negative patients with primary biliary cirrhosis using recombinant autoantigens. Hepatology 2001;34:243-8.

13. Angulo P, Maor-Kendler Y, Lindor KD. Small-duct primary sclerosing cholangitis: a long-term follow-up study. Hepatology 2002;35:1494-500.

14. Burak K, Angulo P, Pasha TM, Egan K, Petz J, Lindor KD. Incidence and risk factors for cholangiocarcinoma in primary sclerosing cholangitis. Am J Gastroenterol 2004;99:523-6.

15. Kim WR, Therneau TM, Wiesner RH, Poterucha JJ, Benson JT, Malinchoc M, et al. A revised natural history model for primary sclerosing cholangitis. Mayo Clin Proc 2000;75:688-94. 